\section{\$11. Dependence of Radial Thermal Diffusivity on Parameters of Toroidal Plasma Affected by Resonant Magnetic Perturbations}

Kanno, R., Nunami, M., Satake, S., Takamaru, H. (Chubu Univ.), Okamoto, M.* (Chubu Univ.)

Using a $\delta f$ drift-kinetic simulation code, we investigate how the radial thermal diffusivity of an axisymmetric toroidal plasma is altered by effect of resonant magnetic perturbations (RMPs) in a low collisionality regime $\nu_{\text {eff }}<\omega_{\mathrm{b}}$, where $\nu_{\text {eff }} \sim \nu / \epsilon_{\mathrm{t}}$ is the effective collision frequency, $\nu$ is the collision frequency, $\epsilon_{\mathrm{t}}$ is the inverse aspect ratio, and $\omega_{\mathrm{b}}$ is the bounce frequency. The perturbed region is assumed to be generated on and near the resonance surfaces, and is wedged in between the regular closed magnetic surfaces. Dependence of the thermal diffusivity on parameters of the plasma is investigated in the simulations ignoring the electric field and the mean velocity. The simulation conditions are as simplified as possible for the sake of visible prospect of the investigation. It is found that the thermal diffusivity is altered as $\chi_{r}=\chi_{r}^{(0)}\left\{1+c_{0}\left(\omega_{\mathrm{b}}\left|B_{\mathrm{to} 0}\right|^{2} / \nu_{\mathrm{eff}} m\right)\left\langle\left\|\delta B_{r}\right\|^{2}\right\rangle /\left|B_{\mathrm{to}}\right|^{2}\right\}$. The thermal diffusivity evaluated in the simulations is extremely small compared with that predicted by the theory of field-line diffusion (the FLD theory). ${ }^{1)}$ Here, $\chi_{r}^{(0)}$ is the neoclassical thermal diffusivity, $\left\langle\left\|\delta B_{r}\right\|^{2}\right\rangle^{1 / 2}=$ $\left\langle\sum_{k, \ell}\left|\delta B_{r}^{(k / \ell)}\right|^{2}\right\rangle^{1 / 2}$ is the strength of the RMPs in the radial directions, $\left|\delta B_{r}^{(k / \ell)}\right|$ is the strength of the RMP causing resonance with a rational surface of $q=k / \ell, q$ is the safety factor, $\langle\cdot\rangle$ means the flux surface average defined with the unperturbed magnetic field, $\left|B_{\mathrm{t} 0}\right|=B_{\mathrm{ax}}$ is the strength of the unperturbed magnetic field on the magnetic axis, $m$ is the particle mass, and $c_{0}$ is a positive coefficient which is independent of $\left\langle\left\|\delta B_{r}\right\|^{2}\right\rangle^{1 / 2}, \nu$, $m$ and $\left|B_{\mathrm{to}}\right|$. The fact remains that the coefficient $c_{0}$ is undefined in the present study, and it will be a topic in the future study. In the case that $\nu_{\mathrm{eff}}>\omega_{\mathrm{b}}$, we find that $\chi_{r}$ evaluated in the simulations becomes close to $\chi_{r}^{(0)}$.

Applying the drift kinetic equation solver KEATS ${ }^{2)}$ to the heat transport of ion (proton) in a circular tokamak field disturbed partly by RMPs, we evaluate the radial thermal diffusivity from the radial heat flux given by a guiding center distribution function $f=f_{\mathrm{M}}+\delta f$. Here, the Maxwell distribution $f_{\mathrm{M}}$ is assumed to be given as a function of $r$ and $v, r$ is the label of the original flux surfaces, $v$ is the speed of an ion, the density and temperature, $n$ and $T$, are fixed in the simulations, and the perturbed magnetic field is also fixed. We find that the thermal diffusivity is proportional to $\left\langle\left\|\delta B_{r}\right\|^{2}\right\rangle$, and that this tendency does not change if the collision frequency $\nu$ is changed. Here, $\left|B_{\mathrm{t} 0}\right|=4 \mathrm{~T}, T=1 \mathrm{keV}$, $q \approx 1.45$, and $R_{\mathrm{ax}}=3.6 \mathrm{~m} \mathrm{(} R_{\mathrm{ax}}$ is the major radius of the axis). Then the thermal diffusivity is represented as $\chi_{r}=\chi_{r}^{(0)}\left\{1+c\left\langle\left\|\delta B_{r}\right\|^{2}\right\rangle\right\}$, where $c$ is a positive coefficient. It is shown in figure 1 that the difference between the thermal diffusivities with and without the RMPs is negligibly small in the plateau regime $\nu_{\text {eff }}>\omega_{\mathrm{b}}$, and that one of the key parameters explaining the coefficient $c$ is $\omega_{\mathrm{b}} / \nu_{\text {eff }}$. We also find that $(y / z) \propto\left|B_{\mathrm{t} 0}\right|^{2} / m$, where $y=\left\{\chi_{r} / \chi_{r}^{(0)}\right\}-1$ and $z=\left(\omega_{\mathrm{b}} / \nu_{\mathrm{eff}}\right)\left\langle\left\|\delta B_{r}\right\|^{2}\right\rangle /\left|B_{\mathrm{to}}\right|^{2}$. Consequently, the thermal diffusivity is represented as

$$
\chi_{r}=\chi_{r}^{(0)}\left\{1+\tilde{c}_{0}\left(\frac{q R_{\mathrm{ax}}}{\sqrt{\epsilon_{\mathrm{t}}} \Delta_{\mathrm{b}}}\right)^{2}\left(\frac{\omega_{\mathrm{b}}}{\nu_{\mathrm{eff}}}\right) \frac{\left\langle\left\|\delta B_{r}\right\|^{2}\right\rangle}{\left|B_{\mathrm{t} 0}\right|^{2}}\right\},
$$

where $\Delta_{\mathrm{b}}$ is the width of a banana orbit and $\tilde{c}_{0}=$ $\left(\Delta_{\mathrm{b}}^{2}\left|B_{\mathrm{t} 0}\right|^{2} / q^{2} R_{\mathrm{ax}}^{2} m\right) c_{0}$ is a positive coefficient.

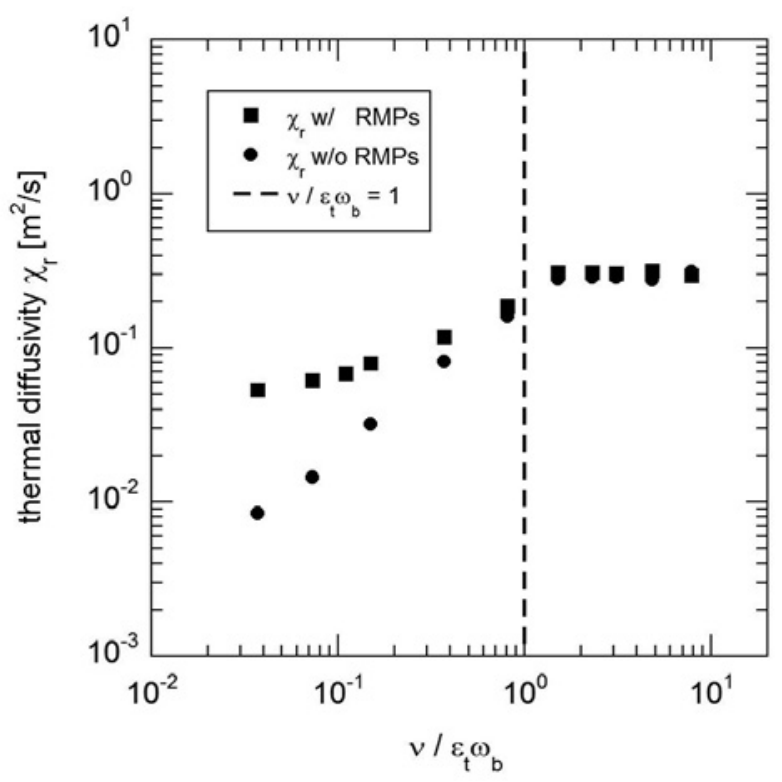

Fig. 1: The thermal diffusivity depends on $\omega_{\mathrm{b}} / \nu_{\mathrm{eff}}$ in the case with RMPs (squares) / without RMPs (circles).

If the ergodic region is generated, the thermal diffusivity evaluated in the simulations is extremely small compared with that predicted by the FLD theory. This tendency is consistent with the recent experimental results. ${ }^{3}$ ) The thermal diffusivity in the perturbed region is expected to be affected by boundary conditions on the edge of the perturbed region, and the boundary conditions will be studied in the near future.

1) Rechester A B and Rosenbluth M N 1978 Phys. Rev. Lett. 4038.

2) Kanno R et al 2010 Plasma Phys. Control. Fusion 52 115004

3) Evans T E et al 2006 Nature Phys. 2419.

*Deceased. 